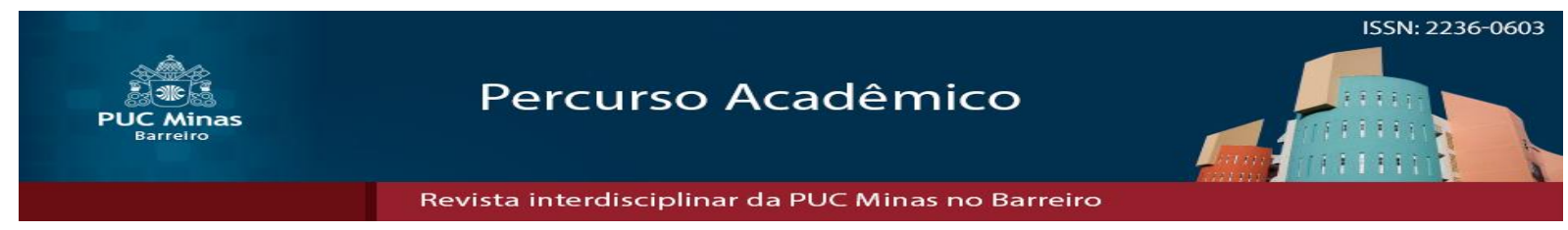

\title{
A percepção de acadêmicos de enfermagem quanto aos usuários de drogas: uma revisão de literatura
}

\section{The perception of nursing students regarding drug users: a literature review}

\author{
Deborah Costa Esquarcio ${ }^{1}$ \\ Quésia Nayrane Ferreira ${ }^{2}$ \\ Juliana Alves de Jesus ${ }^{3}$
}

\begin{abstract}
Resumo
O abuso de drogas é um tema relevante na saúde pública, agravado pelo despreparo dos profissionais. Trata-se de uma revisão da literatura com o objetivo de apreender o que há referente à concepção e percepção do acadêmico de enfermagem sobre usuários de drogas. Utilizou-se quatro artigos que versam sobre o assunto e que foram publicados no período de 2008 a 2016, com a utilização dos descritores Enfermagem, Drogadição e Reabilitação na Biblioteca Virtual em Saúde (BVS). Os resultados apontaram para uma concepção, por parte dos estudantes, próxima ao senso comum quanto às drogas, o que revela uma lacuna na formação do profissional enfermeiro. O enfermeiro presta assistência direta aos usuários, familiares e comunidade, construindo-se, assim, um profissional que deve ser preparado adequadamente em sua graduação para o atendimento a esse público específico. Dessa forma, a inclusão da temática das drogas na formação acadêmica se faz necessária para a formulação de novos conhecimentos.
\end{abstract}

Palavras-chave: Enfermagem. Drogadição. Reabilitação.

\begin{abstract}
Drug abuse is a relevant issue in public health, aggravated by the unpreparedness of professionals. This is a review of the literature with the objective of understanding what is related to the conception and perception of nursing students about drug users. Four articles that deal with this issue were published in the period between 2008 and 2016, using the descriptors Nursing, Drogadition and Rehabilitation in the Virtual Health Library (VHL). The results pointed to a conception on the part of the students close to the common sense as the drugs, which reveals a gap in the formation of the professional nurse. The nurse provides direct assistance to the users, family and community, thus building a professional who must be properly prepared in his graduation to attend this specific public. In this way, the inclusion of the drug theme in academic formation is necessary for the formulation of new knowledge.
\end{abstract}

Keywords: Nursing. Substance-Related Disorders. Rehabilitation

\footnotetext{
Artigo recebido em 08 de Junho de 2016 e aprovado em 28 de Junho de 2017.

${ }^{1}$ Enfermeira - UCMG, Habilitação em Enfermagem em Saúde Pública - UCMG, Psicóloga clínica - PUC MG Especialista em Psicoterapia Fenomenológico Existencial, Mestre em Psicologia Social - UFMG. E-mail: dsquarci@ gmail.com.

${ }^{2}$ Enfermeira graduada pela Pontifícia Universidade Católica de Minas Gerais- PUC MG. E-mail: quesiaferreira20@gmail.com.

${ }^{3}$ Enfermeira graduada pela Pontifícia Universidade Católica de Minas Gerais- PUC MG. E-mail: ju.alves2@yahoo.com.br.
} 



\section{Introdução}

O fenômeno do uso e abuso indistinto de drogas na sociedade contemporânea aponta para uma relevante questão de saúde pública que emerge mundo afora. Para alguns, a drogadição se revela como uma possibilidade de ir ao encontro da felicidade, para outros é a possibilidade de alívio frente à condição existente e, para todos nós, é um processo irremediável do mundo atual que afeta a todos com desconfortáveis indagações.

O último relatório do Escritório das Nações Unidas contra Drogas e Crime (UNODC) 2015 estima que 246 milhões de pessoas, ou seja, cerca de 5\% da população global, entre 15 e 66 anos, usou drogas ilícitas no ano de 2013. E que, nesse mesmo ano, foram registradas 187.100 mortes prematuras pelo abuso de substâncias. São 27 milhões de pessoas que fazem o uso problemático de drogas, sendo quase a metade de drogas injetáveis. (UNODC, 2015).

Os homens são três vezes mais propensos ao uso de maconha, cocaína e anfetamina, enquanto as mulheres são mais propensas a usar incorretamente opióides de prescrição e tranquilizantes. Ocorreu uma diminuição do uso de cocaína, mas o uso de maconha e o uso medicinal de opióides da indústria farmacêutica continuam em ascensão, sendo que o consumo de heroína e ópio permanece inalterado de maneira global. (UNODC, 2015).

São muitas a consequências para a saúde pública mundial, acarretadas pelo uso e abuso de drogas identificadas nas infecções por hepatites virais, HIV/AIDS, desnutrição, comprometimento da saúde bucal, transtornos psiquiátricos e outros. (SENAD, 2009).

Foram identificadas 541 novas substancias psicoativas até o final de 2014 que geram impactos negativos a saúde em 95 países e territórios. Mas há evidências de que mais pessoas estão sendo afetadas com as consequências do uso da maconha e que, provavelmente, a maconha vem se tornando mais prejudicial, pois há um significativo número de pessoas buscando primeiro tratamento em diversas partes do mundo. (UNODC, 2015).

De forma geral, a procura por tratamento sofreu significativa alta por usuários de estimulantes à base de anfetamina, inclusive metanfetamina e MDMA ou ecstasy, bem como para recentes substâncias psicoativas que são denominadas "drogas legais". (UNODC, 2015).

De acordo com o Centro Brasileiro de Informações sobre Drogas Psicotrópicas CEBRID (2015), do Departamento de Medicina Preventiva da Universidade Federal de São Paulo (UNIFESP), a Organização Mundial de Saúde - OMS estabelece critérios gerando a seguinte classificação: uso na vida - pelo menos uma vez nos últimos 30 dias; uso no ano uso de drogas pelo menos uma vez no último ano; uso recente ou no mês - uso de droga pelo 
menos uma vez no último mês; uso frequente - uso de droga seis ou mais vezes no último mês; uso de risco - uso que implica alto risco de dano à saúde, ainda não resultou em doença física ou mental; e o uso prejudicial - uso que está causando dano à saúde física ou mental.

O ultimo Relatório Brasileiro sobre Drogas é de 2009 e objetiva analisar e reportar, no período de 2001 à 2007, a situação da demanda, da oferta e dos danos relacionados ao consumo de drogas no Brasil, e é uma produção da Secretaria Nacional de Políticas sobre Drogas. (SENAD, 2009).

Não é possível estabelecer uma comparação com o relatório da UNODC porque os dados brasileiros estão dispostos considerando estudos epidemiológicos distintos quanto a levantamentos domiciliares sobre o uso de drogas psicotrópicas, levantamento nacional sobre os padrões de consumo de álcool na população brasileira e de álcool e outras drogas na população indígena, além de outros aspectos abordados.

No entanto, o relatório revela que o uso de drogas na vida dos brasileiros, com exceção de álcool e tabaco, aumentou de 19,4\% em 2001 para 22,8\% em 2005. Também aumentaram o consumo de álcool, tabaco, maconha, solventes, benzodiazepínicos, cocaína, estimulantes, esteróides, alucinógenos e crack na classificação uso na vida. Os dados apontam para uma redução de anticolinérgicos (chás e substâncias sintéticas), cujo uso contínuo causa desinteresse pela vida e desorientação. (SENAD, 2009).

É ainda notável a identificação de que nos homens há maior uso na vida e também maior dependência de álcool em comparação com as mulheres em todas as faixas etárias. A faixa etária na qual se situa a maior dependência de álcool é entre 18 a 24 anos e, na sequência, de 25 a 35 anos. As mulheres apresentam uma percepção do risco grave no consumo de álcool, maconha, cocaína e crack mais ressaltado que os homens e, possivelmente, esse fator explique o consumo em maior frequência e intensidade por eles. (SENAD, 2009).

É a esse sujeito, que necessita de um acompanhamento adequado e constante, que o acadêmico de enfermagem presta atendimento no exercício de suas práticas de estágio, e essa formação irá impactar em breve na sua atuação como enfermeiro, propiciando ou não o desenvolvimento de práticas adequadas de promoção à saúde e prevenção de agravos quanto à dependência química.

A relevância deste artigo se dá pela identificação da escassez na literatura de trabalhos envolvendo os conceitos de concepção e percepção de estudantes de enfermagem sobre usuários de drogas. A formulação adequada desses conceitos, enquanto graduandos em 
enfermagem, é imprescindível no exercício da boa prática profissional. Assim, o objetivo deste estudo foi realizar uma revisão bibliográfica quanto à concepção de acadêmicos de enfermagem sobre usuários de drogas.

\section{Metodologia}

Este artigo é uma revisão integrativa da literatura com o objetivo de reunir e resumir o conhecimento científico já produzido sobre o tema, o que possibilitou buscar, avaliar e sintetizar os estudos disponíveis para contribuir com o desenvolvimento do conhecimento na temática. Foram percorridas etapas na elaboração do estudo como: definição dos objetivos e do problema de pesquisa; estabelecidos os critérios de inclusão de trabalhos; busca da literatura; análise com o uso de categorização dos achados e discussão dos resultados. (MENDES; SILVEIRA; GALVÃO, 2008).

Partiu-se do tema enfermagem junto aos usuários de álcool e outras drogas no processo de reabilitação. Para as buscas utilizou-se da combinação dos seguintes descritores: enfermagem, drogadição, dependência química e reabilitação. Essa ocorreu no período de março de 2016 na Biblioteca Virtual em Saúde (BVS). Optou-se por essa base por entender que é uma coleção de fonte de dados que atinge a literatura latino-americana em enfermagem com a publicação de periódicos conceituados da área da saúde.

Os resumos foram avaliados, sendo selecionados 12 artigos para serem lidos na íntegra. Como critérios de inclusão foram utilizados artigos publicados no período de 2008 a 2016, disponíveis em texto completo e que, pelo resumo, possibilitaram estabelecer alguma relação com o profissional enfermeiro e o usuário de droga.

Após a leitura dos trabalhos selecionados na íntegra, chegou-se a conclusão de que o tema posteriormente definido foi contemplado em apenas uma das publicações selecionadas, mas que as leituras revelaram um novo assunto de grande relevância: a concepção dos acadêmicos de enfermagem sobre usuários de drogas. Com essa mudança de tema, foram realizadas novas pesquisas na BVS com a combinação dos descritores acadêmico e estudante com os descritores utilizados na primeira pesquisa (enfermagem, drogadição, dependência química e reabilitação) sem resultados diferentes.

A quantidade de trabalhos publicados quanto à concepção e percepção do acadêmico de enfermagem frente ao fenômeno do uso e abuso de drogas permitiu a seleção de quatro artigos, aqueles que versam pela concepção do acadêmico de enfermagem. Prosseguiu-se com a análise e organização das categorias temáticas na construção dos resultados e discussões. 


\section{Resultados e Discussões}

Com a intenção de identificar e analisar a concepção dos acadêmicos de Enfermagem sobre os usuários de drogas, foram avaliados quatro artigos científicos que atenderam aos critérios previamente estabelecidos.

Referindo-se ao delineamento, três artigos tiveram abordagem qualitativa, sendo um com a aplicação de grupo focal, outro com entrevistas individuais e outro com utilização da pesquisa-ação. Um dos artigos menciona ser descritivo exploratório e utilizou como instrumento de coleta uma Escala de Atitudes e Crenças - NEADA (Nursing Education in Alcool and Drug Education) que é parte de um programa de treinamento para enfermeiros, estudantes e docentes de Enfermagem de Connecticut, EUA.

Quanto ao ano e idioma de publicação, verificou-se que o período variou de 2008 a 2015, sendo dois em português, um em inglês e um em espanhol. A origem das pesquisas foi Guayaquil no Equador, Argentina e duas no Brasil, sendo uma nas regiões do Grande Rio, Serrana, Norte e Sul Fluminense, e outra na cidade de Teresina.

Em um dos artigos, para caracterizar o termo "concepção", utilizou-se de MichaelisModerno Dicionário da Língua Portuguesa (2008), como "imagem subjetiva do mundo, concebida por um individuo ou grupo, de acordo com determinado ponto de vista". Recorrendo a esta exposição, depreenderam que a constituição da concepção se dá "a partir de conhecimentos com contribuições de crenças individuais construídas a partir de culturas distintas". (LOPES et al. 2009).

No mesmo artigo, na sequência, utilizou-se da formulação do conceito de crença como "algo aceito como verdadeiro pelo julgamento da probabilidade, ao invés de sê-lo pela sua realidade". Portanto, o que fica evidente nas condutas é marcadamente mais assentado no elemento "fé do que em um fato". As crenças pessoais podem revelar veracidade ou ainda apresentar demonstrações de confiabilidade, gerando a experiência de autenticidade. (LOPES et al. 2009).

A construção desses conceitos se faz necessária para a afirmação de que as concepções que são oriundas das crenças, via de regra, marginalizam o usuário de drogas devido a um ajuizamento de valor que poderá induzir o profissional de saúde a uma abordagem preconceituosa e a atitudes inadequadas, comprometendo eticamente a assistência prestada. (LOPES et al. 2009). 
Observou-se que em ambos os artigos existe a referência a escassez de conhecimento quanto à dependência de drogas. E, assim, pode-se inferir o mesmo resultado de que citações são baseadas em informações do senso comum que foram apreendidas por meios de comunicação e pelo conhecimento adquirido de diferentes formas por cada sujeito envolvido no estudo, o que revelou a lacuna na abordagem do assunto na academia. (CASTELO BRANCO; MONTEIRO; VARGAS, 2015).

Em Lopes et al. (2009), estabeleceu-se conexões entre a formação do enfermeiro e a assistência prestada ao paciente usuário de drogas, afirmando haver um descompasso de conhecimento, uma vez que a grade curricular de grande parte das instituições de ensino superior não considera conteúdos necessários ao exercício profissional frente a complexa questão de uso e abuso de substâncias psicoativas. Afirmam ainda que, esse hiato de conhecimento estende-se aos docentes que não estão preparados para lidar com a temática das drogas e que esse despreparo tem perpetuado na formação de novos profissionais.

Ao tratar o conceito das drogas, por parte dos estudantes de enfermagem, os resultados demonstraram que eram consistentes com a abordagem científica da literatura, mas as citações se deram pela concepção que tinham adquirido como um "senso comum" pela maioria. Esses abordavam as drogas como substâncias que causam mudanças físicas e comportamentais e que essas alterações representam um problema social e econômico que não afeta apenas o usuário, mas a familia e comunidade. O mesmo se deu quanto ao conhecimento sobre as políticas atuais referentes às drogas. (CASTELO BRANCO; MONTERIO; VARGAS, 2015).

$\mathrm{Na}$ apropriação de conhecimentos significativos na universidade, os alunos contam com grandes iniciativas para realizarem intervenções na abordagem de alguns conteúdos na prática, mas, posteriormente, não dão continuidade na aplicação das intervenções. Essas intervenções oferecidas não abrangem o conteúdo da promoção à saúde incorporada frente à dependência de drogas, e sim ao tratamento do consumidor. (ROJO; BUENO; SILVA, 2008).

A academia oferece abordagens de diversas disciplinas que tentam, assim como a enfermagem, compreender o homem, para formar um marco de trabalho interdisciplinar e intersetorial. Entretanto, à medida que a interdisciplinaridade avança, aparecem, cada vez mais, novos desafios.

Segundo Lindo (2003), a universidade é considerada uma instituição social, responsável pelo desenvolvimento de um alto nível acadêmico dos recursos humanos. Assim sendo, essa deve formar e preparar profissionais para que consigam enfrentar os crescentes desafios atuais com êxito, nas esferas científica, técnica e cultural. 
De acordo com Rojo; Bueno; Silva (2008), os profissionais da saúde são capazes de promover competências cognitivas e metacognitivas, apoderar-se de saberes socialmente válidos e reproduzi-los, no contexto do campo de atuação em sua prática profissional. Os autores destacam que as concepções dos estudantes sobre o contato com o paciente possibilitam pôr em prática os conhecimentos de promoção à saúde, para sua futura atuação profissional, articulação teoria-prática, e possuem um papel fundamental no desenvolvimento das experiências dos estudantes.

Os estudantes têm uma percepção depurada da realidade e é indispensável trabalhar com esses acadêmicos sobre "ações preventivas e promoção da saúde, pilares fundamentais da atenção primária em saúde e os problemas de saúde da alta prevalência”. (BERMÚDEZHERRERA et al., 2011).

Em Rojo; Bueno; Silva (2008) foram extraídas concepções dos acadêmicos sobre a promoção à saúde e a dependência de drogas. Três categorias distintas de análises puderam ser extraídas do discurso dos integrantes dos grupos focais. Primeira categoria - enfermagem e promoção da saúde: aplicação no futuro exercício profissional. Segunda categoria - o fenômeno das drogas: apreciações e crenças sobre o uso de substâncias psicoativas. Terceira categoria - um olhar crítico sob a realidade dos estudantes e a aproximação de conhecimentos significativos na universidade.

Em Bermúdez-Herrera et al. (2011), a percepção dos estudantes sobre o que leva um indivíduo ao abuso de drogas produziu cinco categorias, sendo uma sobre a situação econômica gerando desemprego e imigração, bem como a ausência de uma política econômica e social que atue na prevenção do consumo de drogas, além da fragilidade das leis estabelecidas. A segunda é a violência intrafamiliar revelada na agressão física e psicológica sofrida por mulheres e crianças de seus pais drogados, produzindo nos jovens a procura de alívio no uso de drogas. Seguida pela imigração ou disfunção de familiares próximos e pais de crianças e adolescentes que ficam sob a responsabilidade de tios e avós. Essas buscam suporte em seus pares que os indicam o caminho do consumo de drogas.

A quarta categoria é a influência do meio como "a discriminação, o abandono, a desorganização da família e os antecedentes familiares de consumo de álcool e drogas" que atuam como mola propulsora na busca pelas drogas para abrandar o sofrimento. E, por último, o desconhecimento do tema, pois os próprios estudantes não sabem informar sobre instituições para o tratamento ou prevenção do consumo de drogas. (BERMÚDEZHERRERA et al., 2011). 
Os acadêmicos de enfermagem ressaltam a grande importância da promoção antiuso de substâncias psicoativas para a sociedade. Os estudantes têm como concepção que as classes sociais, ao sustentar que a pobreza, constituem um fator que predispõe o sujeito ao consumo dessas substâncias, permitem-lhe evadir-se da realidade. E que, nas ações de promoção da saúde, os acadêmicos têm o professor como mediador desse processo que necessita de um conhecimento especifico (ROJO; BUENO; SILVA, 2008; CASTELO BRANCO; MONTERIO; VARGAS, 2015)

Em Rojo; Bueno; Silva (2008), os modelos médicos de atendimento foram destacados como dificultador para planejar ações de promoção à saúde nos cuidados da enfermagem, já que são centrados na cura e reabilitação, prevalecendo na prática médica. Salientam que, no ciclo acadêmico, dentro da universidade de medicina, o enfoque prevalente é o biológico, mesmo o modelo médico tendo uma bagagem teórica. No entanto, apenas alguns profissionais estão direcionados à promoção da saúde frente ao uso de substâncias psicoativas. Os acadêmicos consideram que o foco na cura e reabilitação interfere nas ações do enfermeiro, quanto à prevenção.

A concepção dos acadêmicos traz uma breve referência ao modelo cultural, enfatizando a autoestima e a importância que a família e a escola possuem. (ROJO; BUENO; SILVA, 2008). Também que as diferentes formas de composição familiar são importantes no tratamento e reabilitação, mas não é uma responsabilidade de forma isolada. (CASTELO BRANCO; MONTERIO; VARGAS, 2015). E, segundo Oliveira e Mendonça (2012), o papel da família é mais importante sob a perspectiva de prevenção e tratamento da dependência química.

Em Rojo; Bueno; Silva (2008), foi identificado que a maior resistência na comunicação entre enfermeiro e paciente diz respeito à aversão moral. Nos resultados, evidenciou-se que a maioria dos acadêmicos considera desconfortável para o paciente ser indagado sobre o uso de álcool e drogas. Acreditam que gera irritabilidade nos pacientes o fato de serem indagados sobre assuntos de cunho pessoal, como uso e abuso de drogas. E não acreditam nas respostas dos usuários quando indagados pelo enfermeiro sobre consumo. Esses achados invocam a carência de mais esforços serem empreendidos na formação acadêmica quanto à questão de álcool e drogas, por ser o profissional enfermeiro, via de regra, o que estabelece o primeiro contato com o paciente.

O diálogo tende a ser favorável entre acadêmicos e usuários de drogas, mas também se 
destaca a inquietação quanto ao número significativo de acadêmicos cujas concepções dificultam a acessibilidade do usuário, impossibilitando o estabelecimento de uma relação favorável com os pacientes. (LOPES et al., 2009).

Há menção sobre alterações que as drogas acarretam à saúde dos usuários, sendo a maioria voltada para mudanças de comportamento, perda cognitiva e outros fatores de risco que contribuem para o desenvolvimento de doenças oportunistas. Abordam o "mundo das drogas" como facilitador da aquisição de Doenças Sexualmente Transmissíveis (DST) e de transtornos mentais, como alucinações e delírios, que desencadeiam o comportamento agressivo dos usuários. (CASTELO BRANCO; MONTERIO; VARGAS, 2015).

Relatam ainda que a busca pela aquisição da droga é que leva o dependente a infringir a lei, envolvendo-se em diversos atos de violência, e ao isolamento social. Isso porque o consumo se torna cada vez mais intenso, colocando o usuário em situação de fragilidade rompendo as relações emocionais e os vínculos constituídos entre familiares, amigos e a sociedade de forma geral. (CASTELO BRANCO; MONTERIO; VARGAS, 2015).

\section{Considerações finais}

O fenômeno da drogadição requer serviços e profissionais estruturados para a atenção integral ao usuário, família e comunidade, visto que afeta a todos de forma direta ou indireta. Sendo o enfermeiro um profissional fundamental nesse processo faz-se necessária a abordagem deste tema desde a academia, preparando-os para o mercado de trabalho.

Quanto à percepção dos estudantes de enfermagem, esta revisão destaca que depende muito do que é apreendido culturalmente sobre drogas e que a abordagem desse conteúdo na universidade depende da interação do grupo docente com o assunto, gerando uma lacuna na formação dos acadêmicos pelo fato desses professores revelarem baixo conhecimento do assunto. A evidência da escassez de conhecimento dos estudantes sobre a dependência de drogas reforça que as apreciações são superficiais e impregnadas de conhecimento intuitivo.

As evidências encontradas nas literaturas estudadas apontam para a necessidade de promoção à saúde, quanto ao antiuso de drogas e a importância do enfermeiro em planejar ações de promoção e cuidados perante aos usuários de drogas ilícitas, desde o ciclo acadêmico, para um futuro exercício profissional.

Dessa forma, faz-se necessária a formulação de um currículo acadêmico com a obrigatoriedade de se abordar esse assunto com os estudantes de enfermagem. Os alarmantes números referentes à dependência química reforçam, cada vez mais, a necessidade de 
profissionais capacitados no mercado.

Além disso, é fundamental que sejam realizados novos estudos na busca de produção de conhecimento na área, para que haja mudanças de condutas na atuação dos profissionais de saúde na assistência aos usuários e na luta contra o uso abusivo de substâncias psicoativas. 


\section{REFERÊNCIAS}

BERMUDEZ-HERRERA, Azucena et al. Percepción de los estudiantes de una escuela de enfermería acerca del consumo de drogas lícitas e ilícitas. Rev. Latino-Am. Enfermagem, RibeirãoPreto, v. 19, p.684-690, Jun. 2011.

CASTELO BRANCO, Fernanda Matos Fernandes; MONTEIRO, Claudete Ferreira de Souza; VARGAS, Divane de. Knowledge of nursing students about drugs and drug control policies. Revista de Pesquisa: Cuidado é Fundamental Online, [S.1.], v. 7, n. 2, p.2215-2228, Abr. 2015.

CEBRID. Centro Brasileiro de Informações sobre Drogas Psicotrópicas. UNIFESPUniversidade Federal de São Paulo, 2015. Disponível em:

<http://www.cebrid.epm.br/index.php>. Acesso em: 09 abr. 2015

LINDO, Augusto Pérez. Universidad, conocimiento y reconstrucción nacional. Buenos Aires: Biblos, 2003. 4 v. ( Colección Educación y sociedad.)

LOPES, Gertrudes Teixeira; LEMOS, Bruna Kelly de Jesus; LIMA, Helen Balthazar de; CORDEIRO, Bárbara Rodrigues Carvalho; LIMA, Luana dos Santos Vasconcellos.

Concepções de acadêmicos de enfermagem sobre usuários de drogas. Rev. Bras. Enferm., Brasília, v. 62, n. 4, p.518-523, Ago. 2009.

MENDES, Karina Dal Sasso; SILVEIRA, Renata Cristina de Campos Pereira; GALVÃO, Cristina Maria. Revisão integrativa: método de pesquisa para a incorporação de evidências na saúde e na enfermagem. Texto Contexto - Enferm., Florianópolis , v. 17, n. 4, p.758-764, Dez. 2008

OLIVEIRA, Elias Barbosa, MENDONÇA, Jovana Lucia Schettini. Familiar com dependência química e consequente sobrecarga sofrida pela família. Online Brazilian Journal of Nursing, v. 11, n. 1, Mai. 2012.

ROJO, Maria Della; BUENO, Sonia Maria Villela; SILVA, Edilaine Cristina da. Conceptions of nursing students on health promotion related to psychoactive substances. Rev. Latino-Am. Enfermagem, Ribeirão Preto, v. 16, p.627-633, Aug. 2008.

SENAD. Secretaria Nacional de Políticas sobre Drogas. Relatório Brasileiro sobre Drogas 2009. Disponível em: <www.senad.org.br >. Acesso em: 09 abr. 2016.

UNODC - Escritório das Nações Unidas sobre Drogas e Crime. World Drug Report 2015. Disponível em: <www.unodc.org >. Acesso em: 09 abr. 2016. 